Bull. Austral. Math. Soc.

11E12, 11F30

VOL. 65 (2002) [231-238]

\title{
AN EXPLICIT HECKE'S BOUND AND EXCEPTIONS OF EVEN UNIMODULAR QUADRATIC FORMS
}

\author{
KoK SEng ChuA
}

\begin{abstract}
We prove an explicit Hecke's bound for the Fourier coefficients of holomorphic cusp forms for $S L_{2}(Z)$ and apply it to derive effectively computable constants $c(m)$ for each dimension $m$, divisible by 8 , for which every even integer is always represented by every even unimodular form of $m$ variables.
\end{abstract}

\section{INTRODUCTION}

Let $f\left(x_{1}, \ldots, \dot{x}_{m}\right)$ be an even unimodular positive definite quadratic form in $m$ variables. (This implies $m$ is a multiple of 8.) An even integer $a$ is said to be exceptional for $f$ if $f$ does not represent $a$ for integral $\left(x_{1}, \ldots, x_{m}\right)$. A theorem of Tartakovsky [8] imples that $a$ is not exceptional if it is sufficiently large. More exactly, for each dimension $m$ divisible by 8 , there is a constant $c(m)$ such that $a \geqslant c(m)$ implies that $a$ is represented by all $f$ of $m$ variables. In [6], Peters initiated a method for finding explicit values for $c(m)$ and he computed some values of $c(m)$ for $m \leqslant 64$. This was improved and extended first to $m \leqslant 72$ by Odlyzko and Sloane [5] and later by Charaborty, Lai and Ramakrishnan [1] and Lai and Ramakrishnan [3] up to $m \leqslant 184$ by essentially transforming Peter'sidea to one of linear programming. However it seems to us that an explicit value of $c(m)$ which works for all $m$ has still not been given.

In this note we shall give one such explicit bound based on a modification of Peters' idea. His method in [6] is based on the theory of modular forms and especially an effective Deligne's estimate for the Fourier coefficients of eigenforms (Ramanujan-Petersson conjecture). Peters remarked that his method would work in principle in any dimensions but it gets laborious as soon as the dimension of the space of cusp forms grow bigger than two. The main problem with the method is that to use an effective form of Deligne's estimate, one needs to first obtain a basis of eigenforms which are hard to compute. Our first idea is to replace Deligne's estimate by an effective Hecke's bound which is weaker but more general and will avoid the need to compute eigenforms. Hecke's bound is

$$
a_{n}=O\left(n^{k / 2}\right)
$$

Received 7th August, 2001

Copyright Clearance Centre, Inc. Serial-fee code: 0004-9727/02 \$A2.00+0.00. 
where $a_{n}$ is the $n$-th Fourier coefficient for a cusp form of weight $k$. In (1) there is an implied constant which depends on the modular form which we need to make explicit. Our first result is an effective and explicit version of (1). Even though weak asymptotically, it may be of some independent interest for small values of $n$. We have the following.

THEOREM 1. Let $f(z)=\sum_{n=1}^{\infty} a_{n} q^{n} \in S_{k}$, the space of holomorphic cusp forms of weight $k$ for $S L_{2}(Z)$ and let $s$ be the dimension of $S_{k}$. Then we have for each $n=1,2,3, \ldots$ the estimate

$$
\left|a_{n}\right| \leqslant\left(C_{k} \sqrt{\sum_{j=1}^{s}\left|a_{j}\right|^{2}}\right) n^{k / 2},
$$

where $C_{k}$ is a constant depending only on $k$ which may be taken as

$$
C_{k}=e^{2 \pi} \sqrt{\lambda_{k}} \operatorname{Sup}_{z \in H}\left(\sqrt{\sum_{j=1}^{s}\left|f_{j, k}(z)\right|^{2} y^{k}}\right)
$$

and $f_{j, k}(z)=\sum_{z=0}^{\infty} a_{j, k}(n) q^{n}=\Delta^{j}(z) E_{k-12 j}(z), \Delta(z)$ being the unique cusp form of weight 12 and $E_{j}(z)$ is the normalised Eisenstein series of weight $j$ (with $E_{0}(z)=1$ ) and the supremum is taken over the upper half plane $H$, and $\lambda_{k}$ is the maximum eigenvalue of $\left(H_{k} H_{k}^{T}\right)^{-1}, H_{k}$ being the $s$ by $s$ matrix given by (10) below constructed from the initial $s$ coefficients $\left\{a_{j, k}(n)\right\}$ of $f_{j, k}(z)$.

From Theorem 1 we obtain immediately our main result.

THEOREM 2. Let $f\left(x_{1}, \ldots, x_{m}\right)$ be an even unimodular quadratic form in $m=2 k$ variables. Then with the notation of Theorem $1, f$ represents $2 n$ provided

$$
n>\left(\frac{e^{2 \pi} \zeta(k) \Gamma(k)}{(2 \pi)^{k}} \alpha_{k} \sqrt{\lambda_{k}} \operatorname{Sup}_{z \in H} \sqrt{\sum_{j=1}^{s}\left|f_{j, k}(z)\right|^{2} y^{k}}\right)^{2 /(k-2)},
$$

where $\alpha_{k}=\sqrt{\sum_{j=1}^{s}\left|a_{j}\right|^{2}}$ and $a_{j}$ are constants depending on $f$ which may be bounded uniformly above using the crude estimate $\left|a_{j}\right| \leqslant \max \left\{4 j(2 j+1)^{4 k},(2 \pi j)^{k} /(j \Gamma(k))\right\}$ for $j=1, \ldots, s$.

REMARK. The $a_{j}$ are the coefficients of a cusp form which is the difference between the theta series of $f$ and the Eisenstein series of weight $k$ (see the proof of Theorem 2 below).

We shall prove our main results (Theorem 1 and 2) in Section 2. In Section 3 we give explicit computable bounds for the right hand side of (4) and give numerical values $c(m)$ for $m \leqslant 192$ above which an even integer is not exceptional for any even unimodular form in $m$ variables. 


\section{Proof of Main Results}

Let $M_{k}$ (respectively $S_{k}$ ) be the space of holomorphic modular forms (respectively cusp forms) of weight $k$. Let $s$ be the dimension of $S_{k}$ so that $\operatorname{dim}\left(M_{k}\right)=s+1$. It is well know that

$$
s= \begin{cases}{\left[\frac{k}{12}\right]} & k \neq 2 \bmod 12 \\ {\left[\frac{k}{12}\right]-1} & k=2 \bmod 12\end{cases}
$$

Since the space of cusp forms has dimension $s$, it is reasonable to expect that an explicit form of (1) should take the form

$$
\left|a_{n}\right| \leqslant M\left(a_{1}, \ldots, a_{s}\right) n^{k / 2}
$$

and in order to prove an estimate of form (6), one needs a way to express every cusp form in terms of its first $s$ coefficients. This can be done explicitly if one chooses a standard basis "supported on $\{1, \ldots, s\}$ " in a sense. Indeed there is always one such integral basis as proven in Lang [4, Chapter X, Theorem 4.4] and [1, Lemma A.1].

Lemma 2.1. There is a basis $\left\{g_{j, k}(z)=\sum_{n=0}^{\infty} g_{j, k}(n) q^{n}: 0 \leqslant j \leqslant s\right\}$ for $M_{k}$ such that the coefficients $g_{j, k}(n)$ are all integral and $g_{j, k}(n)=\delta_{j, n}$ for all $0 \leqslant j, n \leqslant s$. It follows that any $f(z)=\sum_{n=0}^{\infty} a_{n} q^{n}$ in $M_{k}$ can be expressed uniquely as a linear combination $f(z)=\sum_{j=0}^{s} a_{j} g_{j, k}(z)$ and if $a_{0}, \ldots, a_{s}$ are integral so are all the $a_{n}$. In particular the $g_{j, k}(z)$ are themselves unique and have integral coefficients. The same holds for the cusp forms $\left\{g_{1, k}, \ldots, g_{s, k}\right\}$ as a standard basis for $S_{k}$.

By the uniqueness of the standard basis in Lemma 2.1, we can compute explicitly their Fourier coefficients in a uniform way. Let $\Delta(z)=\sum_{n=1}^{\infty} \tau(n) q^{n}$ be the unique cusp form of weight 12 and $E_{j}(z)=1+A_{j} \sum_{n=1}^{\infty} \sigma_{j-1}(n) q^{n}$ be the normalised Eisenstein series of weight $j$. Here $\tau(n)$ is the Ramanujan function, and

$$
A_{j}=(-1)^{j / 2}(2 j) / B_{j / 2}=(-1)^{j / 2} \frac{(2 \pi)^{j}}{\zeta(j) \Gamma(j)}
$$

and $\sigma_{j-1}(n)$ is the sum of the $(j-1)$ th power of the divisor of $n$. The coefficients of $\left\{g_{j, k}(z)\right\}$ can be expressed explicitly in terms of $\tau(n)$ and $\sigma_{j}(n)$ as follows. Let

$$
f_{j, k}(z)=\Delta^{j}(z) E_{k-12 j}(z)=\sum_{n=0}^{\infty} a_{j, k}(n) q^{n} \quad 0 \leqslant j \leqslant s .
$$


The coefficients $\left\{a_{j k}(n)\right\}$ can be explicity computed to be

$$
a_{j, k}(n)= \begin{cases}0 & n<j \\ 1 & n=j \\ \tau_{j}(n)+A_{k-12 j} \sum_{i=j}^{n-1} \tau_{j}(t) \sigma_{k-12 j-1}(n-t) & n>j\end{cases}
$$

where the $\tau_{j}(n)$ are coefficients of $\Delta^{j}(z)=\sum_{n=j}^{\infty} \tau_{j}(n) q^{n}$. Note that the entries are rational.

LEMMA 2.2. Let $H_{k}$ be the $s$ by $s$ upper triangular matrix given by

$$
H_{k}=\left(\begin{array}{cccccc}
1 & a_{1, k}(2) & \ldots & \ldots & \ldots & a_{1, k}(s) \\
& 1 & a_{2, k}(3) & \ldots & \ldots & a_{2, k}(s) \\
& & \ddots & \vdots & \vdots & \vdots \\
& & & 1 & \ldots & a_{s-2, k}(s) \\
& & & & 1 & a_{s-1, k}(s) \\
& & & & & 1
\end{array}\right)
$$

where $\left\{a_{j, k}(i)\right\}$ are defined explicity by (9), then we have

$$
\left(\begin{array}{c}
g_{1, k}(z) \\
\vdots \\
g_{s, k}(z)
\end{array}\right)=H_{k}^{-1}\left(\begin{array}{c}
f_{1, k}(z) \\
\vdots \\
f_{s, k}(z)
\end{array}\right)
$$

Proof of LemMa 2.2: By Lemma 2.1, we have

$$
f_{j, k}(z)=\sum_{i=0}^{s} a_{j, k}(i) g_{i, k}(z) \quad \text { for } 0 \leqslant j \leqslant s
$$

which gives us (11) when we delete the first equation.

REMARK. By taking the $n$th coefficients in (11), we obtain an explicit formula for $\left\{g_{j, k}(n)\right\}_{1 \leqslant j \leqslant s}$ in terms of $\left\{a_{j, k}(n)\right\}$. A similar formula for $\left\{g_{0, k}(n)\right\}$ can be obtained from (12). We note incidentally that this gives explicit formulae for the coefficients of the theta series $g_{0, k}(z)$ of the extremal lattice in dimension $2 k$.

We can now derive an estimate for our basis forms.

LEMMA 2.3. For all $z \in H$, the upper half plane, we have

$$
\sum_{j=1}^{s}\left|g_{j, k}(z)\right|^{2} \leqslant \lambda_{k} \sum_{j=1}^{s}\left|f_{j, k}(z)\right|^{2}
$$

where $\lambda_{k}$ is the maximum eigenvalue of $\left(H_{k} H_{k}^{T}\right)^{-1}$ and $H_{k}$ is defined as in (10). 
ProOF OF LeMMA 2.3: By Lemma $2.2(11)$, we have $\sum_{j=1}^{s}\left|g_{j, k}(z)\right|^{2}=Q(v, \bar{v})$ where $Q$ is the positive definite quadratic form given by the symmetric matrix $\left(H_{k} H_{k}^{T}\right)^{-1}$ and $v$ is the vector $\left(f_{1, k}(z), \ldots, f_{s, k}(z)\right)^{T}$. Clearly $Q(v, \bar{v}) \leqslant \lambda_{k} \bar{v}^{T} v$.

LEMMA 2.4: Let $N_{2 i}$ be the number of representations of $2 i$ by an even quadratic form, in $m=2 k$ variables, then $\sum_{i=1}^{j} N_{2 i} \leqslant 4 j(2 j+1)^{k}$.

Proof of LemMA 2.4: It is more convenient to use the language of lattices. We follow an argument of Conway [2, p. 332]. Let $u, v$ be lattice vectors in an $m=2 k$ dimensional lattice $L$ with $u \neq \pm v$ and $\|u\|^{2},\|v\|^{2} \leqslant 2 j$, and suppose they belong to the same class in $L / \lambda L$. If $\lambda^{2} \geqslant 2 j$ then $2 \lambda^{2} \leqslant\|\lambda w\|^{2}=\|u-v\|^{2}=4 j-2\langle u, v\rangle \leqslant 4 j$, where we replace $v$ by $-v$ if ncessary. We must therefore have equality everywhere and $\langle u, v\rangle=0$. It follows that $\sum_{i=1}^{j-1} N_{2 i}+\left(N_{2 j} / 2 m\right) \leqslant \lambda^{2 k}$ and the Lemma follows.

Proof ofThEOREM 1: By a standard argument using the invariance of $|f(z)|^{2} y^{k}$ and the residue formula as in, for example Serre [7, Chapter VII, Theorem 5], we have

$$
\left|a_{n}\right| \leqslant e^{2 \pi} M n^{k / 2}
$$

where

$$
M=\operatorname{Sup}_{z \in H}\left(|f(z)| y^{k / 2}\right)
$$

By Lemma 2.1, we have $f(z)=\sum_{j=1}^{s} a_{j} g_{j, k}(z)$, so

$$
|f(z)|^{2} \leqslant \sum_{j=1}^{s}\left|a_{j}\right|^{2} \sum_{j=1}^{s}\left|g_{j, k}(z)\right|^{2} \leqslant \sum_{j=1}^{s}\left|a_{j}\right|^{2} \lambda_{k} \sum_{j=1}^{s}\left|f_{j, k}(z)\right|^{2}
$$

by Lemma 2.3. (2) and (3) follow immediately from (14), (15) and the last inequality. REMARK. We note that the supremum is finite since one need only maximise over the fundamental domain by invariance and the fact that $|f(z)| y^{k / 2} \rightarrow 0$ as $y \rightarrow i \infty$.

Proof of THEOREM 2: By a result of Hecke, the theta series of $f, \theta_{f}(z)=$ $\sum_{x \in Z^{m}} q^{f(x)}=\sum_{n=0}^{\infty} N_{2 n} q^{n} \in M_{k}$ for $k=m / 2$ where $N_{2 n}$ is the number of times $2 n$ is represented by $f$. Clearly $\theta_{f}(z)=E_{k}(z)+h(z)$ where $h(z)=\sum_{n=1}^{\infty} a_{n} q^{n} \in S_{k}$. It follows that

$$
N_{2 n}=A_{k} \sigma_{k-1}(n)+a_{n}
$$

So $N_{2 n}$ cannot be zero if $\left|a_{n}\right|<A_{k / 2} \sigma_{k-1}(n)$, or using (2) if $C_{k} \sqrt{\sum_{j=1}^{s}\left|a_{j}\right|^{2}} / n^{k / 2-1}<A_{k / 2}$ (we have used the fact that $\left(\sigma_{k-1}(n)\right) / n^{k-1}=\sum_{d \mid n}\left(1 / d^{k-1}\right)>1$ ). This gives the sufficient 
condition

$$
n>\left(\frac{C_{k} \sqrt{\sum_{j=1}^{s}\left|a_{j}\right|^{2}}}{A_{k / 2}}\right)^{2 /(k-2)}
$$

and this is just (4) on substituting the value of $C_{k}$ from (3) if we set $\alpha_{k}$ to be $\sqrt{\sum_{j=1}^{s}\left|a_{j}\right|^{2}}$.
The upper bound for $\left|a_{j}\right|$ follows from (16), Lemma 2.4, and that

$$
A_{k / 2} \sigma_{k-1}(j)=\frac{(2 \pi)^{k}}{\zeta(k) \Gamma(k)} j^{k-1} \sum_{d \mid j} \frac{1}{d^{k-1}} \leqslant \frac{(2 \pi j)^{k}}{j \Gamma(k)} \frac{\zeta(k-1)}{\zeta(k)} \leqslant \frac{(2 \pi j)^{k}}{j \Gamma(k)} .
$$

\section{EXPLiCIT ESTIMATES}

In order to give explicit values $c(m)$ beyond which an even integer is not exceptional for a quadratic form in $m$ variables, we must give explicit computable bounds for the terms $\alpha_{k}, \lambda_{k}$ and the supremum occuring on the right side of Theorem 2. These are given in the three lemmas below.

LEMMA 3.1. $\left|a_{j}\right| \leqslant \max \left\{4 j(2 j+1)^{k},(2 \pi j)^{k} / j \Gamma(k)\right\}$. This has already been observed in the Proof of Theorem 2.

LEMMA 3.2. Let $H_{k}^{-1}=\left(h_{i j}\right)$ and $\lambda_{k}$ be the maximum eigenvalue of $\left(H_{k} H_{k}^{T}\right)^{-1}$, then $\lambda_{k} \leqslant \sum_{i, j}\left|h_{i j}\right|^{2}$.

PROOF OF LEMMA 3.2: This follows from the general fact that for any real matrix $A\left(=H_{k}^{-1}=\left(h_{i j}\right)\right)$, the maximum eigenvalue of $A^{T} A$ is equal to the operator norm $\|A\|$ which is trivially bounded above the Frobenius norm $\|A\|_{F}=\sum_{i} \sum_{j}\left|h_{i j}\right|^{2}$.

LEMMA 3.3. Let $f(x)=\sum_{n=1}^{\infty} n^{6} x^{n-1}$ and $f_{j}(x)=\sum_{n=1}^{\infty} n^{j} x^{n}$ which are convergent for $|x|<1$, then for $j=1,2, \ldots$

$$
\left|\Delta^{j} E_{k-12 j}(z)\right| y^{k / 2} \leqslant\left(\sqrt{3} f\left(e^{-\pi \sqrt{3}}\right)\right)^{j}\left(\frac{k}{4 \pi j e}\right)^{k / 2}\left[1+\frac{(2 \pi)^{k-12 j} f_{k-12 j-1}\left(e^{-\pi \sqrt{3}}\right)}{\Gamma(k-12 j)}\right] .
$$

Proof of LEMMA 3.3: By invariance we only need to prove a bound for $z$ in a fundamental domain so we may assume $y \geqslant \sqrt{3} / 2$. We split the left hand side into three parts,

$$
\left|\frac{\Delta(z)}{e^{-2 \pi y}}\right|^{j}\left|y^{k / 2} e^{-2 \pi j y}\right|\left|E_{k-12 j}(z)\right|=I . I I . I I I \text {. }
$$

For I, we have $\left|\Delta(z) / e^{-2 \pi y}\right| \leqslant \sum_{n=1}^{\infty}|\tau(n)| e^{2 \pi(n-1) y}$. Using Delinge's estimate that $|\tau(n)| \leqslant$ $d(n) n^{11 / 2}$ and that $|d(n)| \leqslant \sqrt{3 n}$ gives the first term in the bound of (19). The bound for II is just calculus and the bound for III follows from

$$
\left|E_{j}(z)\right| \leqslant 1+\frac{(2 \pi)^{j} \zeta(j-1)}{\Gamma(j) \zeta(j)} \sum_{n=1}^{\infty} n^{j-1} e^{-2 \pi n y} \leqslant 1+\frac{(2 \pi)^{j}}{\Gamma(j)} f_{j-1}\left(e^{-2 \pi y}\right)
$$




\begin{tabular}{|c|c|c|c|c|c|}
\hline $\mathrm{M}$ & $\mathrm{c}(\mathrm{m})$ & $\mathrm{m}$ & $\mathrm{c}(\mathrm{m})$ & $\mathrm{m}$ & $\mathrm{c}(\mathrm{m})$ \\
\hline 24 & 169 & 80 & 25081 & 136 & 418771 \\
\hline 32 & 249 & 88 & 49688 & 144 & 619999 \\
\hline 40 & 992 & 96 & 90217 & 152 & 644199 \\
\hline 48 & 3603 & 104 & 92012 & 160 & 928589 \\
\hline 56 & 4194 & 112 & 161847 & 176 & 1345330 \\
\hline 64 & 10405 & 120 & 259764 & 184 & 1842966 \\
\hline 72 & 23378 & 128 & 270168 & 192 & 2470014 \\
\hline
\end{tabular}

Table 1.

where we have used

$$
\frac{\sigma_{j-1}(n)}{n^{j-1}}=\sum_{d \mid n} \frac{1}{d^{j-1}} \leqslant \zeta(j-1)
$$

An explicit (though somewhat crude) upper bound $c(m)$ can now be computed for (4) using Lemma 3.1-3.3. Explicitly, we have the right hand side of (4) bounded above by

$$
\left(\frac{e^{2 \pi} \zeta(k) \Gamma(k)}{(2 \pi)^{k}} \sqrt{\sum_{j=1}^{s}\left|a_{j}\right|^{2} \sum_{i, j}\left|h_{i j}\right|^{2} \sum_{j=1}^{s} f_{j k}^{2}}\right)^{2 /(k-2)}
$$

where we may replace $a_{j}$ by the righ hand side of Lemma $3.1, f_{j k}$ by the right hand side of (19) and the $h_{i j}$ computed explicitly by inverting the matrix in (10) with the entries given explicitly by (9).

In this way it is easy to obtain the values of $c(m)$ as given in Table 1 above though some care must be taken to arrange the terms so that the numbers do not grow bigger than that representable by the computer. Not surprisingly the values are inferior to those obtained in [2] using Deligne's sharp estimte. However Theorem 2 now gives an explicit $c(m)$ for all $m$.

\section{REFERENCES}

[1] K. Chakraborty, A.K. Lal and B. Ramakrishnan, 'Modular forms which behave like theta series', Math. Comp. 66 (1997), 1169-1183.

[2] J.H. Conway and N.J.A. Sloane, Sphere packings, lattices and groups, Grundlehren der Mathematischen Wissenschaften 290, (second edition) (Springer-Verlag, Berlin, Heidelberg, New York, 1988).

[3] A.K. Lal and K. Chakraborty, 'On exceptions of integral quadratic forms', Contemp. Math. 210 (1988), 151-170.

[4] S. Lang, Introduction to modular forms, Grundlehren der Mathematischen Wissenschaften 222 (Springer-Verlag, Berlin, Heidelberg, New York, 1976). 
[5] A.M. Odlyzko and N.J.A. Sloane, 'On exceptions of integral quadratic forms', J. Reine Angew. Math. 321 (1981), 212-216.

[6] M. Peters, 'Exceptions of integral quadratic forms', J. Reine Angew. Math. 314 (1980), 196-199.

[7] J.P. Serre, $A$ course in arithmetic, Graduate Texts in Mathematics 7 (Springer-Verlag, Berlin, Heidelberg, New York, 1973).

[8] V.A. Tartakovsky, 'Die Gesamtheit der Zahlen, die durch eine positive quadratishe form $F\left(x_{1}, \ldots, x_{s}\right)(s \geqslant 4)$ darstellbar sind', Izv. Akad. Nauk SSSR 7 (1929), 111-122, 165-195.

Institute of High Performance Computing

High End Computing Division

1 Scenic Park Rd

\#01-01 The Capricorn

Singapore Science Park II

Singapore 117528

e-mail: chuaks@ihpc.nus.edu.sg 